\title{
Optical and Structural Studies on SnS Films Grown by Co-Evaporation
}

\author{
C. Cifuentes ${ }^{1}$, M. Botero ${ }^{1}$, E. Romero ${ }^{2}$, C. Calderón ${ }^{1}$, and G. Gordillo ${ }^{1}$ \\ ${ }^{1}$ Departamento de Física, Universidad Nacional de Colombia, Bogotá, Colombia \\ ${ }^{2}$ Departamento de Química, Universidad Nacional de Colombia, Bogotá, Colombia
}

Received on 8 December, 2005

\begin{abstract}
Polycrystalline SnS thin films were grown on glass substrates using a novel procedure involving a chemical reaction between the precursor species evaporated simultaneously. This is a relatively new material, which exhibits excellent properties to be used as absorbent layer in solar cells. X-ray diffraction (XRD) measurements indicate that the synthesized samples grow in several phases $\left(\mathrm{SnS}, \mathrm{SnS}_{2}\right.$ and $\left.\mathrm{Sn}_{2} \mathrm{~S}_{3}\right)$ depending upon the deposition conditions. However, through an exhaustive parameter study, conditions were found to grow thin films predominantly in the $\mathrm{SnS}$ phase with orthorhombic structure. It was found that this type of compound presents p-type conductivity, a high absorption coefficient (greater than $10^{4} \mathrm{~cm}^{-1}$ ) and an energy band gap $E_{g}$ of about $1.3 \mathrm{eV}$, indicating that this compound has good properties to perform as absorbent layer in thin film solar cells.
\end{abstract}

Keywords: SnS; Thin films; Solar cells; Optical properties; Phases

\section{INTRODUCTION}

The most extensively studied polycrystalline thin film solar cells are based on CuInSe 2 (CIS) and CdTe as absorbent material [1]. Modules have been produced using these materials, with efficiencies exceeding $12 \%[2,3]$. Although high efficiency solar cells based on CIS and CdTe have been produced, it is generally recognized that other materials may in the long term have advantages over the former. In the case of CdTe technology, there is concern respect to the use of cadmium and in the case of CIS a major concern is the supply of indium when the technology move to large-scale production; also the toxicity of selenium. Current efforts are being made in various laboratories to develop new photovoltaic materials to improve the efficiency without these problems. The factors that should be considered in developing new photovoltaic materials include: a suitable energy band gap, the possibility of depositing the material using low cost deposition methods, abundance of the precursor elements and low environmental costs with respect to the synthesis of the elements, production, operation and disposal of modules. Tin sulphide $(\mathrm{SnS})$ is one of the promising materials for low cost thin film solar cells technology, because of its optimum energy bang gap $E_{g}$ and a high fundamental absorption coefficient [4]. Elements $\mathrm{Sn}$ and $\mathrm{S}$ are abundant in nature; moreover, they do not contribute to pollution during the $\mathrm{SnS}$ growing process.

SnS thin films have been deposited by different techniques such as: the vacuum evaporation of the $\mathrm{SnS}$ compound [5], two stage process [6] and electrochemical deposition [7]. In this work, thin films of $\mathrm{SnS}$ have been grown by coevaporation of the precursor species. A description of the methods used for the growth of the SnS films and details of the effect of the deposition parameters on the optical properties as well as on the phase and crystalline structure in which the samples grow, will be reported.

\section{EXPERIMENTAL}

Thin films of $\mathrm{SnS}$ were prepared by co-evaporation of the precursor species on a glass substrate, heated at temperatures ranging from 200 to $400{ }^{\circ} \mathrm{C}$. Fig. 1 shows the experimental set-up of the system used in the present study.

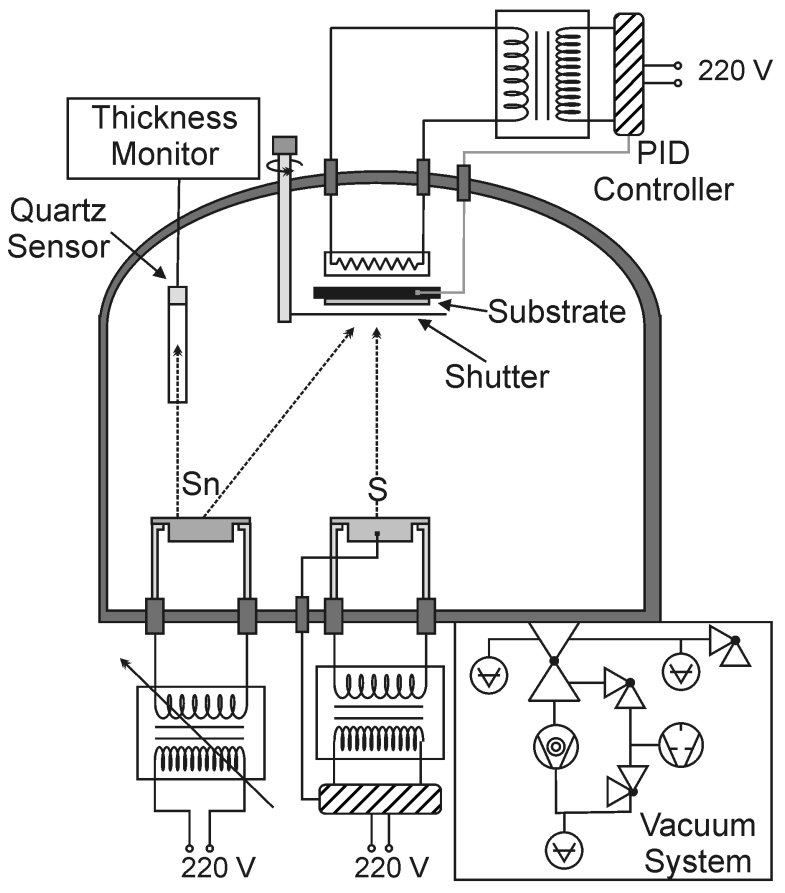

FIG. 1: Experimental set-up used for the deposition of $\mathrm{SnS}$ thin films by co-evaporation of Sn and S.

The deposition system is constituted by the evaporation chamber connected to a vacuum system which allows working at pressures of about $10^{-6}$ Torr , two boats (for the evaporation of S and $\mathrm{Sn}$ respectively) and a thickness monitor (Maxtec TM-400) with a quartz crystal as sensor, used for mea- 
suring the deposition rate of $\mathrm{Sn}$. The substrate temperature is controlled with an Eurotherm 900C PID (proportional integral differential) controller. The deposition rate of $\mathrm{S}$ was not measured because sulfur does not condensate on the quartz crystal under the conditions we grew the $\mathrm{SnS}$ films. The chemical composition of the $\mathrm{SnS}$ films is controlled by controlling the evaporation temperature and/or the mass of evaporated $S$ to evaporated mass of Sn ratio.

In order to find suitable deposition conditions, the $\mathrm{SnS}$ films were prepared varying the deposition parameters in a wide range, as indicated in table $\mathrm{I}$.

The influence of the deposition parameters on the transmittance and on the phase and crystalline structure was determined through spectral transmittance and XRD measurements carried out with a VIS - IR Oriel spectrophotometer and a diffractometer Shimadzu 6000

TABLE I: Variation range of the deposition parameters of SnS thin films deposited be co-evaporation.

\begin{tabular}{|l|l|}
\hline Deposition parameters & Variation range \\
\hline Substrate temperature $\left({ }^{\circ} \mathrm{C}\right)$ & $200-400$ \\
\hline Evaporation Temperat. of S $\left({ }^{\circ} \mathrm{C}\right)$ & $150-170$ \\
\hline Sn-deposition rate $(\AA / \mathrm{s})$ & $0.7-2.5$ \\
\hline Mass of S / mass of $\mathrm{Sn}$ & $2-20$ \\
\hline
\end{tabular}

The optical constants (refractive index $\mathrm{n}$, absorption coefficient $\alpha$, and energy band gap $E_{g}$ ) of the $\mathrm{SnS}$, and $\mathrm{SnS}_{2}$ phases were determined from spectral transmittance measurements and numerical calculations done with the help of a program developed by us. The conductivity type of the SnS films was determined through thermoelectric power measurements.

\section{RESULTS AND DISCUSSION}

\section{A. Structural properties}

A representative number of $\mathrm{SnS}$ films were prepared varying the deposition parameters as indicated in table 1. Each of these samples was initially characterized through transmittance and XRD measurements. From these measurements was obtained information regarding: the phase, crystalline structure, lattice parameters, optical gap and absorption coefficient. These studies showed that, generally, tin sulfide films deposited by co-evaporation, grow with a mixture of the $\mathrm{SnS}$ and $\mathrm{SnS}_{2}$ phases. However, we have also found that using an adequate set of deposition parameters, it is possible to get tin sulfide films in the $\mathrm{SnS}$ phase with orthorhombic structure or in the $\mathrm{SnS}_{2}$ phase with tetragonal structure. The presence of the secondary $\mathrm{Sn}_{2} \mathrm{~S}_{3}$ phase with orthorhombic structure was also identified in several samples.

Most of the deposition parameters affect the phase in which the tin selenide films grow. However, the greatest effect on the chemical composition is caused by the substrate temperature and by the $\mathrm{m}_{S}$ to $\mathrm{m}_{S n}$ ratio. In general, the tin sulfide films tend to grow in the $\mathrm{SnS}$ phase when the substrate tempera- ture is increased and/or when the $\mathrm{m}_{S}$ to $\mathrm{m}_{S n}$ ratio decreases. By the contrary, the $\mathrm{SnS}_{2}$ phase is achieved by decreasing the substrate temperature and/or by increasing the $\mathrm{m}_{S}$ to $\mathrm{m}_{S n}$ ratio. In table II are listed the deposition parameters which allowed to grow tin sulfide films in any of the mentioned phases.

TABLE II: Parameter set through which the $\mathrm{SnS}$ and $\mathrm{SnS}_{2}$ phases are achieved using the co-evaporation method.

\begin{tabular}{|l|l|l|}
\hline \multirow{2}{*}{ Deposition Parameters } & \multicolumn{2}{l|}{} \\
\cline { 2 - 3 } & Shase & \\
\hline Substrate temperature $\left({ }^{\circ} \mathrm{C}\right)$ & 350 & $\mathrm{SnS}_{2}$ \\
\hline Evap. Temperat. of $\mathrm{S}\left({ }^{\circ} \mathrm{C}\right)$ & 180 & 180 \\
\hline Sn-Deposition rate $(\AA / \mathrm{s})$ & 1.8 & 1.8 \\
\hline$\left(\mathrm{m}_{S} / \mathrm{m}_{S n}\right)$ & 5 & 15 \\
\hline
\end{tabular}

Figure 2 shows typical XRD spectra obtained with tin sulfide thin films grown in the $\mathrm{SnS}$ and $\mathrm{SnS}_{2}$ phase respectively. A XRD pattern corresponding to samples presenting a mixture of the $\mathrm{SnS}$ and $\mathrm{SnS}_{2}$ phases is also depicted in Fig. 2.
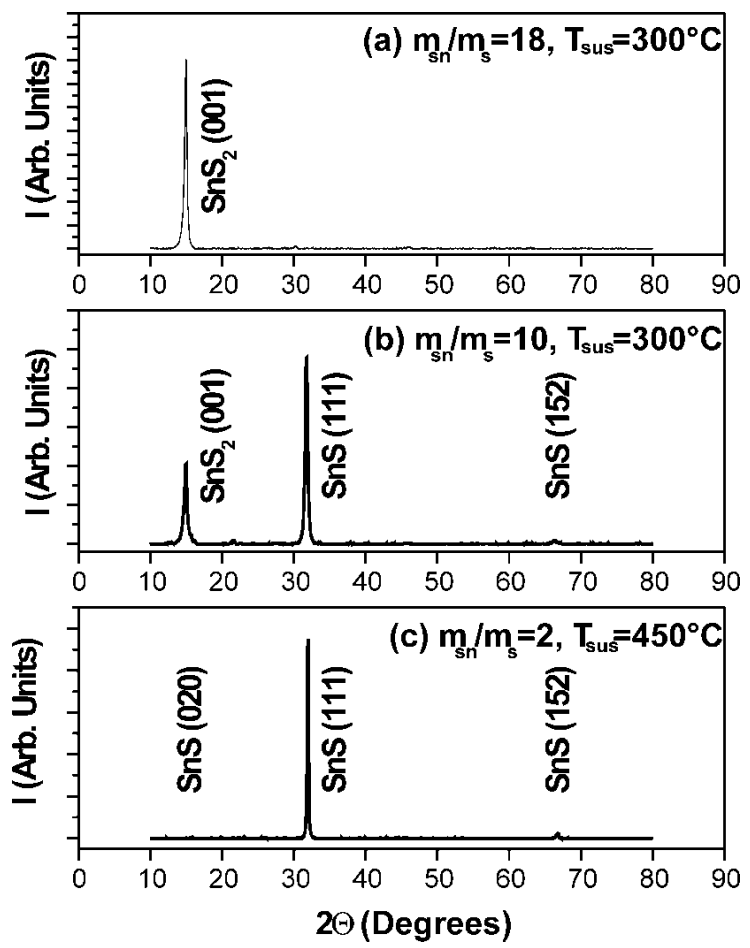

FIG. 2: Typical XRD spectra of tin sulfide thin films grown in: a) $\mathrm{SnS}_{2}$ phase, b) mixture of the $\mathrm{SnS}$ and $\mathrm{SnS}_{2}$ phases and c) $\mathrm{SnS}$ phase.

It was found that the tin sulfide films tend to grow with preferred orientation: the SnS films grow along the (111) plane, whereas the $\mathrm{SnS}_{2}$ films do it along the (001) plane.

Table III contains data regarding structure and lattice constants of typical $\mathrm{SnS}$ and $\mathrm{SnS}_{2}$ thin films deposited by the above mentioned methods; these were obtained comparing the 
TABLE III: Phase, structure and lattice constants of $\mathrm{SnS}$ and $\mathrm{SnS}_{2}$ films prepared by co-evaporation of $\mathrm{S}$ and $\mathrm{Sn}$.

\begin{tabular}{|l|l|l|l|l|}
\hline \multirow{2}{*}{ Phase } & \multirow{2}{*}{ Structure } & \multicolumn{4}{|l|}{ Lattice constant $(\AA)$} \\
\cline { 3 - 5 } & & $\mathrm{a}$ & $\mathrm{b}$ & $\mathrm{c}$ \\
\hline $\mathrm{SnS}^{a}$ & Orthorhombic & 4.101 & 11.205 & 3.861 \\
\hline $\mathrm{SnS}_{2}^{b}$ & Hexagonal & 3.656 & & 5.877 \\
\hline
\end{tabular}

${ }^{a} \mathrm{PDF} 00-039-0354$

${ }^{b}$ PDF\#00-001-1010

XRD pattern of Fig. 2 with the data reported in the JCPDS (Joint Committee on Powder for Diffraction Standards) data base for these types of compounds.

\section{B. Optical properties}

All the deposited $\mathrm{SnS}$ samples were characterized through spectral transmittance measurements. From these measurements was obtained information regarding the influence of the deposition conditions on the optical gap, absorption coefficient and optical constants. In general, each of the deposition parameters affects somehow the transmittance behavior. However, the main features related with the effect the method and preparation conditions cause on the transmittance spectra are the following:

Independently of the phase or structure in which the tin sulfide films grow, their cutoff wavelength $\lambda_{c}$ is significantly influenced by the film thickness d. Fig. 3 shows typical transmittance spectra of $\mathrm{SnS}$ and $\mathrm{SnS}_{2}$ films with different thicknesses. A shift of the transmittance curves toward the region of lower values of $\lambda$ is observed when their thickness decreases.

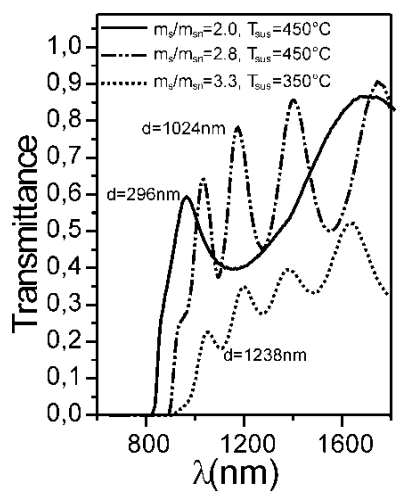

a)

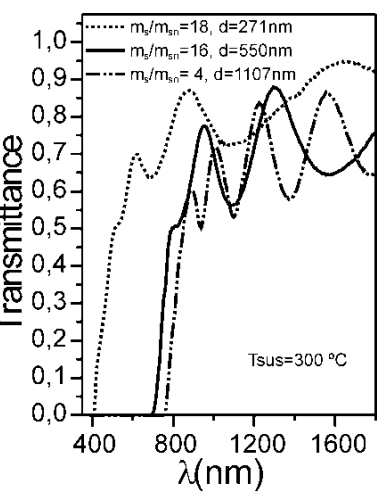

b)
FIG. 3: Influence of film thickness on the spectral transmittance of a) $\mathrm{SnS}$ and b) $\mathrm{SnS}_{2}$ thin films.

We have not found a definitive explanation for this behavior. However, we consider that the shift of the cutoff wavelength observed when the thickness of the $\mathrm{SnS}$ samples in- creases from values smaller than $100 \mathrm{~nm}$, in which the films are in the coalescence stage, up to values of the order of $\mu \mathrm{m}$, in which a continuous film is formed, could be associated to changes in the band structure, induced by changes in the different interaction processes taking place during the different growth stages of the thin films. In particular, the superposition degree of electron clouds of neighboring atoms, which affects the bandwidth, is feasible to increase by increasing the film thickness. It was also found that for thicknesses greater than 1 $\mu \mathrm{m}$, the cutoff wavelength $\lambda_{c}$ of the tin sulfide films keeps approximately constant independently of the film thickness, indicating that the shift of $\lambda_{c}$ can not be associated to an increase of the optical density ( $\alpha . d)$ when the film thickness increases. Therefore, to prevent the effect of the thickness on the finding of the optical gap, samples with thicknesses greater than $1 \mu \mathrm{m}$ will be selected to determine the optical gap, reported for $\mathrm{SnS}$ and $\mathrm{SnS}_{2}$ films respectively.

The cutoff wavelength of the films grown in the $\mathrm{SnS}_{2}$ phase is lower than that of the $\mathrm{SnS}$ films, indicating that the optical gap $E_{g}$ of the $\mathrm{SnS}_{2}$ films is greater than that of the $\mathrm{SnS}$ films. In Fig. 4 are depicted typical transmission spectra of tin sulfide films of similar thicknesses, showing the shift of the transmission spectrum of the SnS films respect to that of the $\mathrm{SnS}_{2}$ films.

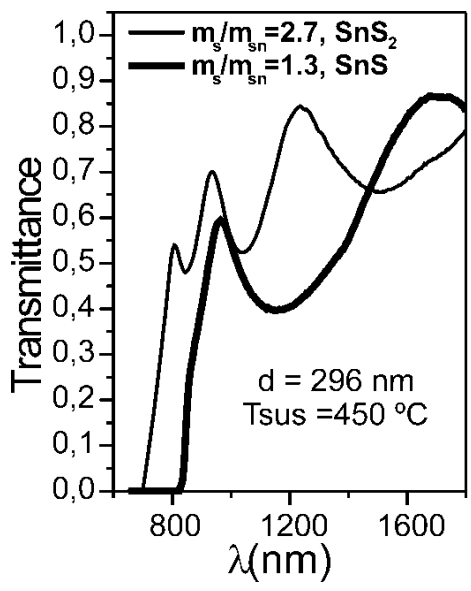

FIG. 4: Comparison of the transmission spectrum of a typical SnS film with a typical $\mathrm{SnS}_{2}$ film.

\section{Optical constants}

This section presents results corresponding to the calculation of the optical constants (refractive index $n$, absorption coefficient $\alpha$ and optical gap $E_{g}$ ) of the $\mathrm{SnS}$ and $\mathrm{SnS}_{2}$ films. These were obtained using the transmittance spectrum of typical films with thicknesses greater than $1 \mu \mathrm{m}$, in order to prevent the effect of the thickness on the band structure.

Figure 5 shows curves of $n$ vs. $\lambda, \alpha$ vs. $\lambda$, and $(\alpha h v)^{2}$ vs. $h v$, obtained for the $\mathrm{SnS}$ and $\mathrm{SnS}_{2}$ films. The optical gap 
$E_{g}$, was determined from the intercept with the hv axis at the linear part of the graph of $(\alpha h v)^{2}$ vs $h v$.

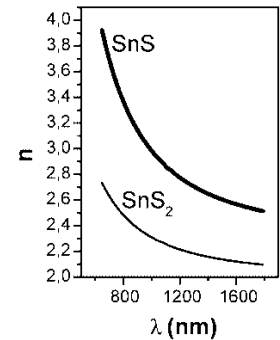

a)

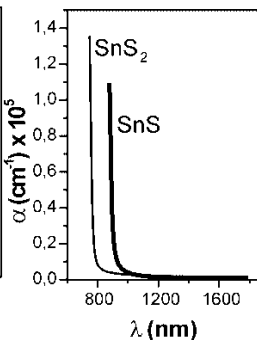

b)

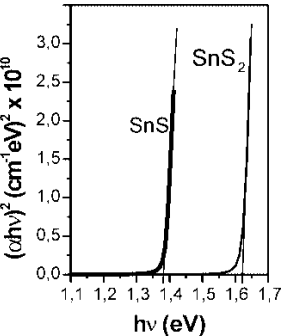

c)
FIG. 5: Curves of a) $n$ vs. $\lambda$, b) $\alpha$ vs. $\lambda$ and c) $(\alpha h v)^{2}$ vs. $h v$ corresponding to $\mathrm{SnS}$ and $\mathrm{SnS}_{2}$ thin films with thicknesses greater than $1 \mu \mathrm{m}$.

From the results shown in Fig. 5, the following facts can be remarked: (i) In the range studied, the refractive index of the $\mathrm{SnS}$ films varies between 2.5 and 3.9; similar values have been reported by other authors [8], for $\mathrm{SnS}$ films deposited by evaporation. On the other hand, it was found that the refractive index of the $\mathrm{SnS}$ films is significantly greater than that of the $\mathrm{SnS}_{2}$ films. This behavior could be attributed to the fact that the SnS films present, generally, an electrical conductivity greater than the $\mathrm{SnS}_{2}$ films, taking into account that the refractive index can be expressed by the relation, $n=4 \pi \sigma / c \alpha$; (ii) The $n$ vs. $\lambda$ curves of the $\mathrm{SnS}$ and $\mathrm{SnS}_{2}$ films, fitted quite well to a polynomial expression of the form $n=\mathrm{A}+\mathrm{B} / \lambda^{2}$ (Cauchy equation), indicating that, in the range of wavelengths studied, the tin sulfide films present normal dispersion; (iii) The absorption coefficient of both types of tin sulfide films is greater than $10^{4} \mathrm{~cm}^{-1}$, indicating that these films are adequate to be used as absorber layer in thin film solar cells.
In this work we have not taken into account light scattering effects caused by surface roughness in the calculation of $\alpha$. However we recommend to include this effect, in order to prevent an apparent increases in the value of $\alpha$. The linear behavior of the ( $\alpha \mathrm{hv})$ vs hv curves indicates that the $\mathrm{SnS}$ and $\mathrm{SnS}_{2}$ thin films have a direct energy band gap. Using the relation $\alpha^{2}=\mathrm{A}\left(E_{g}-h v\right)$, which is valid for this type of materials, $E_{g}$ values of $1.3 \mathrm{eV}$ and $1.6 \mathrm{eV}$ were found, for the $\mathrm{SnS}$ and $\mathrm{SnS}_{2}$ films respectively.

\section{CONCLUSION}

Through an exhaustive parameter study, the conditions to prepare SnS thin films with adequate properties to be used as absorber layer in thin film solar cells were found. The films were deposited by co-evaporation of the precursor species, which is a novel procedure to prepare this type of compounds.

Characterization carried out through XRD measurements indicated that, depending on the preparation conditions, the tin sulfide films may grow in the $\mathrm{SnS}$ phase with orthorhombic structure or in the $\mathrm{SnS}_{2}$ phase with tetragonal structure or with a mixture of both. The presence of the secondary $\mathrm{Sn}_{2} \mathrm{~S}_{3}$ phase with orthorhombic structure was also identified in several samples.

The optical constants (refractive index $n$, absorption coefficient $\alpha$, and optical gap $E_{g}$ ) of $\mathrm{SnS}$ and $\mathrm{SnS}_{2}$ thin films were determined by simple straightforward calculations using only the transmission spectrum. Values of $\alpha$ greater than $10^{4} \mathrm{~cm}^{-1}$ were found for both types of films (for $h v$ greater than $E_{g}$ ); for the optical gap, values around $1.3 \mathrm{eV}$ and $1.6 \mathrm{eV}$ were found for the $\mathrm{SnS}$ and $\mathrm{SnS}_{2}$ films respectively.

\section{Acknowledgements}

This work was supported by Colciencias and Universidad Nacional de Colombia.
[1] B. von Roedem, K. Zweibel, and H.S. Ulall, 31 st IEEE Photovoltaic Specialist Conference and Exhibition, Lake Buena Vista, Florida (2005) 183

[2] W. Diehl, V. Sittinger, and B. Szyszka, Surface \& Coatings Technology 193, 329 (2005).

[3] J.A. M. Abu, S. Johnston, T. Moriarty, G. Teeter, K. Ramanathan, and R. Nuofi, Prog. In Photoovoltaics: Res. Appl. 12, 39 (2004).

[4] M.T. S. Nair, P.K. Nair, Semicond. Sci. Technol.6, 132 (1991).
[5] L. Price, I.P. Parkin, A.M.E. Hardy, and R.J.H Clark, Chem Mater. 11, 1792 (1999).

[6] K.T. Ramakrishna, P. Purandar, Materials Letters 56, 108 (2002).

[7] K. Takeuchi, M. Ichimura, E. Arai, and Y. Yamazaki, Solar Energy Mater. Solar Cells, 75, 427 (2003).

[8] M.M. El-Nahass, H.M. Zeyada, M.S. Aziz, and N.A. ElGhamaz, Optical Materials 20, 159 (2002). 